\title{
Magnetic Resonance Imaging features of breasts in patients with nipple discharge
}

\author{
Shahnaz Choudhury, Ichiro Isomoto and Kuniaki Hyashi \\ Department of Radiology, School of Medicine, Nagasaki University, Nagasaki, Japan.
}

\begin{abstract}
A retrospective study was conducted to demonstrate the potential role of dynamic contrast enhanced magnetic resonance imaging (DCEMRI) in the detection of breast lesions in 15 patients with nipple discharge. The DCEMRI findings were compared and correlated with the findings of ultrasonography and mammography. The results revealed that the character of the nipple discharge was mostly bloody and in few cases were serous. The imaging findings revealed that DCEMRI is highly sensitive and specific in the detection of breast canner and the histopathological correlation in such detection was highly significant $(\mathrm{p}<0.001)$ as compared to ultrasonography and mammography finding.
\end{abstract}

\section{Introduction}

Nipple discharge is the third most common breast complaint for women seeking medical treatment after lump and breast pain ${ }^{1}$. The commonest cause of nipple discharge in non-lactating breast includes papilloma (35-48\%), carcinoma (5-21\%) and duct ectasia (1-3\%). Nipple discharge may be either provoked or spontaneous and unilateral or bilateral. Similarly, nipple discharge may be benign or malignant, serous or sero-sangtinous, while bloody nipple discharge suggests malignancy ${ }^{1}$. The conventional diagnostic imaging of nipple discharge includes mammography, ultrasonography and galactography. But the limitations of these modalities have provoked us to explore alternative imaging modalities, one of which is dynamic contrast enhanced magnetic resonance imaging (DCEMRI). The present study was done to through some lights on this aspect.

\section{Patients and Methods}

A retrospective study was conducted in 15 patients aged between 22 to 71 years (mean 48 years) with nipple discharge. The characteristics of nipple discharge of these patients were mostly bloody and in few cases serous. All these patients had initial magnetic resonance imaging (MRI) examination, then subsequently, had ultrasonography and mammography examination to detect the nature and characteristics of the lesions responsible for nipple discharge.

Mammograms were obtained with dedicated mammogram units (Mammodiagnost, Philip system, Best, Netherlands). Craniocaudal and oblique views were obtained in all patients, if necessary, mediolateral and magnification views were obtained in dedicated mammography cassettes (Min-R 2000; Kodak, Rochester, NY) and screen (Min-R 2000; Kodak).

Ultrasonography was performed with $7.5 \mathrm{MHz}$ hand-held linear transducer (Iogiq 700MR, General Electric Medical System, Milwaukee, WI; Aplico 50, Toshiba Medical System, Tokyo, Japan; pro Sound SSP-5000 ALOKA, Tokyo, Japan; SDU2200, Shimadzu, Kyoto, Japan). Breast MRI examinations were performed with a 1.5-T magnet (Sigma; GE Medical System) using a dedicated surface breast coil. The localizing sequence was followed by a axial fat-suppressed T2-weighted sequence (TR/TE,3000-3100/96-104 mmsec, matrix 256 196-224,2 excitations; fiel of view gradient-echo sequence (TR/TE, 6.7/1.7 msec, flip angle, $20^{\circ}$ one excitation, field of view of 20) was then performed before and four times after the bolus intravenous injection of $0.1 \mathrm{mmol} / \mathrm{L}$ of badopenteate dimeglumine (Magnevist, Berlex, Wayne, NJ) per kg body weight.

Image acquisition started $20 \mathrm{sec}$ after contrast material injection and $20 \mathrm{ml}$ of saline bolus. Images 
were obtained sagittally or coronally with $60 \mathrm{sec}$ interval. Section thickness was 4-6 mm with intersection overlapping using a matrix of $512 \mathrm{x}$ 256 and field of view of $18-22 \mathrm{~cm}$.

Post-contrast or sagittal T1- weighted, fatsuppressed, spolled gradient-echo sequece (TR/TE, 9/2.1 msec; flip angle, $20^{\circ}-30^{\circ}$ excitations, 20-48 sections; slice thickness, 5-7 mm with gapless or with intersection overlapping) were obtained $5 \mathrm{~min}$ after injection of contrast material.

Outcome measures: In mammography, the mass, calcification and architectural distortion were seen. The mass and ductal dilatation were examined in ultrasonography. DCEMRI variables include the mass, ductal spread, intraductal hemorrhage and pattern of enhancement. Histopathology was also carried-out.

\section{Results}

The characters of nipple discharge among 15 patients were bloody in 11 (73.3\%) and serous in 2 (13.3\%) cases. The mammography findings demonstrate the presence of mass in $8 \%$, calcification in 31\% and architectural distortion in 23\% (Table I).

Table I: Findings present using different methods of investigation

\begin{tabular}{lc}
\hline Findings & \% Present \\
\hline Mammography (n=13) & 8 \\
Mass & 31 \\
Calcification & 23 \\
Architectural distortion & \\
Ultrasonography (n=15) & 53 \\
Mass & 33 \\
Ductal dilatation & \\
MRI (n=15) & 53 \\
Mass & 27 \\
Ductal spread & 27 \\
Intraductal hemorrhage & \\
\hline
\end{tabular}

The ultrasonography findings revealed the presence of mass in 53\% and ductal dilatation in 33\% cases. While MRI findings revealed the presence of mass in 53\%, ductal spread $27 \%$ and intraductal hemorrhage in $27 \%$ cases respectively. The ultrasonography diagnosis was breast cancer in $46.6 \%$ cases, mastopathy in $13.3 \%$, papilloma in $6.6 \%$, seroma in $6.6 \%$ and cyst in $6.6 \%$ cases etc. (Figure 1). On the other hand, DCEMRI diagnosed breast cancer in 60\% cases, mastopathy in 6\%, papilloma in $23 \%$ and seroma in $11 \%$ cases.
The histological patterns of the breast lesion in these patients were intraductal carcinoma (66.6\%), ductal cell carcinoma in situ (11.1\%), nonintraductal carcinoma (11.1\%) and intraductal papilloma (11.1\%) cases respectively.

Histopathological correlations were 99.3\% with DCEMRI, 80\% with ultrasonography and 53.8\% in mammography respectively. Finally, the sensitivity of these modalities was $85.71 \%$ in DCEMRI, $75 \%$ with ultrasonography and $50.6 \%$ with mammography. The specificity was $100 \%$ with DCEMRI 85\% with ultrasonography and 75\% with mammography respectively.

\section{Discussion}

At present, mammography is the primary imaging modality used to detect clinically and occult breast lesions including breast cancer. But mammography has several limitations in detecting breast lesions. These include low sensitivity and specificity, inability to detect intraductal lesions etc. These limitations have prompted us to undertake this study using alternative or adjunctive modality like MRI to detect clinically and occult breast lesions in patients with nipple discharge ${ }^{1-2}$.

The potential role of MRI in the diagnosis of breast cancer has been investigated since the late 1970s. These early reports suggest that MRI modality though allows detection of many breast lesions but tissue characterization was found to be limited. However, with the advances in surface coil technology along with new imaging protocols and the use of intravenous contrast gadopenetate i.e. enhanced MRI have permitted improved tissue characterization of many breast lesions including occult breast lesions . Thus, MRI of breast lesions have shown as high as $100 \%$ sensitivity to detect clinically and occult breast lesions. The results of the present study strongly support this. The sensitivity of MRI detected in this study was $87.5 \%$ to $100 \%$ and specificity was $100 \%$ as compared to mammography and ultrasonography. In the present study, the sensitivity and specificity of mammography were $50 \%$ and $80 \%$ respectively. On the other hand, the sensitivity and specificity of ultrasonography in the present study were found to the $75 \%$ and $85.7 \%$ respectively, which were greater than mammography but less than MR imaging. Similar observations were made by other studies $^{1-3}$.

Several studies have also been conducted to correlate the imaging findings of these three modalities with histopathological findings. A 


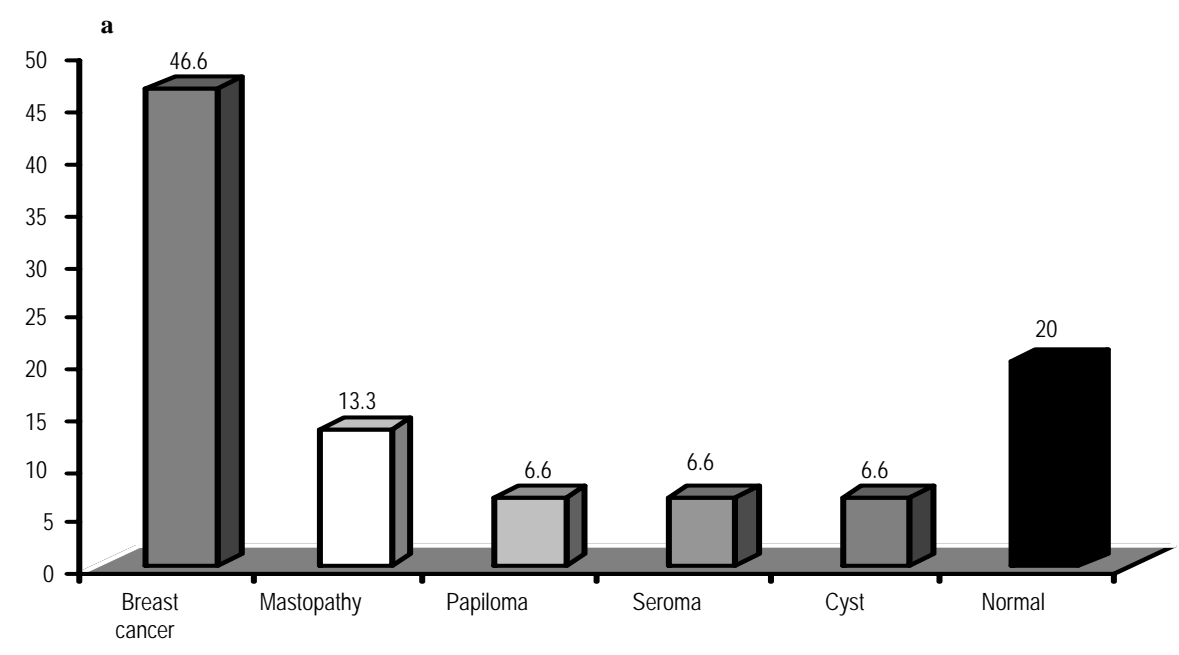

b

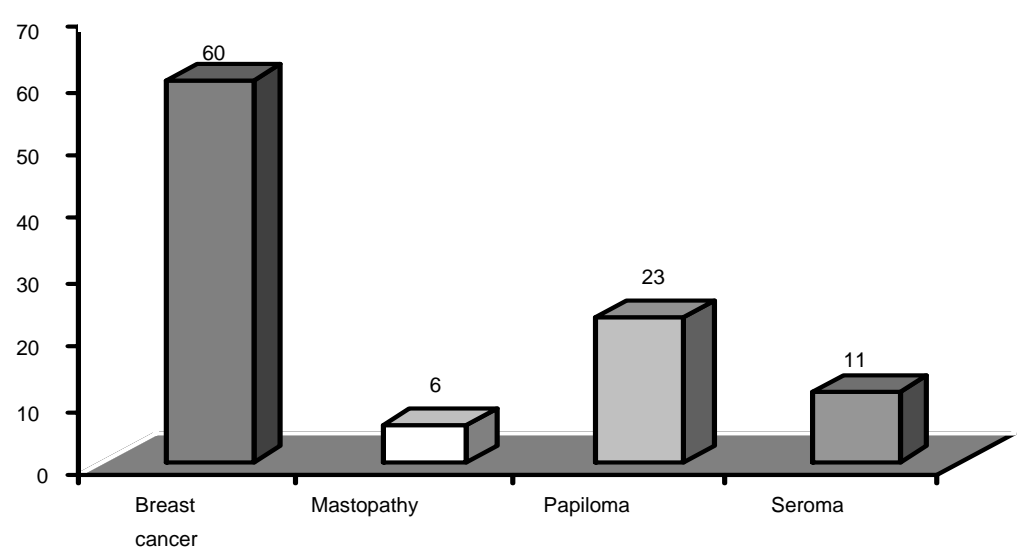

Figure 1: Diagnosis of diseases by a) ultrasonography and b) DCEMRI

number of studies have shown that around $75 \%$ of the MRI correlate well with histopathological findings. In the present series, $99.3 \%$ of contrast enhanced imaging findings correlate with the present study findings. These data strongly support that MRI modatility is highly sensitive and specific in the diagnosis of clinically as well as occult breast lesions.

Furthermore, diagnostic enhanced contrast MRI modality has been found to be useful in the differention of benign from malignant lesions. The observations of this present study strongly support this. In the present study, using dynamic enhanced contrast MRI $60 \%$ of the breast lesions were found to the malignant and $40 \%$ were benign. Similar observations were made by others ${ }^{4-5}$. Moreover, some overlapping between the enhancement of benign and malignant lesions with MRI were found by others leading to a relatively low specificity. However, border characteristics, internal architec- ture, enhancement characteristics and the presence of multiple tiny associated cysts may be the cause of this overlapping. But in the present study, no overlapping was seen in differentiating benign from malignant lesions of breast using MRI modality emphasizing further the specificity of this modality ${ }^{3}$.

Because of high sensitivity and specificity, MRI of the breast offers the best diagnostic options currently available in the diagnosis of clinically and occult breast lesions. The findings of this present study support this ${ }^{1-2}$.

It has also been observed that MRI modality effectively delineates tissue contrast differences and allows the use of intravenous contrast agents that further improves the mass conspicuity ${ }^{4}$

Recent assessments of the potential usefulness and sensitivity of contrast agent enhanced breast MRI have been found to be as high as $94 \%$ to $100 \%$ for 
the detection of breast cancer. The results and observations of the present study with MRI modality closely agree with these observations. In the present study, the sensitivity of MRI modality in the detection of breast lesions are identical to the above observations and we detected sensitivity from $85.7 \%$ to $100 \%$.

There is a growing evidence that MRI can be used to detect underlying mammographically and occult invasive cancer, but little is known about the potential of MRI for detecting mammographically and clinically occult intraductal lesions like ductal carcinoma in situ, papilloma etc. Several studies indicate that in addition to being highly sensitive in the detection of invasive breast cancer, MRI also has the potential and highly sensitive for the detection of intraductal diseases . Thus, MRI would be useful for the detection of breast lesions including intraductal lesions in patients with nipple discharge . The present study also supports this. In this study, $53.3 \%$ of ductal and intraductal lesions, $26.6 \%$ cases hemorrhage in the duct were detected by this modality. Histopathological examinations also correlated well with MRI findings (intraductal cancer \& ductal carcinoma in situ in $66.6 \%$ cases) ${ }^{4}$.

On the other hand, mammography modality has been found including in the present study to be unable to detect any ductal or intraductal lesions. While ultrasonography has been found in the present study to detect abnormality in the duct like ductal dilatation in $33.3 \%$ patients with nipple discharge. It has also been found in some studies that ultrasonography modality, breast cysts was detected in $6.6 \%$ cases. Similarly, both papilloma $(6.6 \%)$ and seroma (6.6\%) cases were detected also in the present study using ultrasonography. But using MRI modality in the present study these masses i.e. papilloma and seroma were detected in higher percentage of cases (23\% for papilloma and $11 \%$ for seroma). Similarly, higher percentage of these cases i.e. papilloma and seroma were also detected by others. On the other hand, using mammography modality, no such cases were detected in the present study.

Nipple discharge while usually benign origin raised concern in both patients and clinicians because of the possibility of an underlying malignancy as the cause. In the present study the character of nipple discharge in majority of the cases (73\%) was bloody and in small number of cases (13.3\%) was serous. But the DCEMRI features revealed $60 \%$ of the breast lesions in the present series are benign. From these results, it can be predicted that the bloody character of nipple discharge observed in the present study could be benign in nature.

\section{References}

1. Orel SG, Dougherty CS, Reynolds C, Czemicki BJ, Siegelman ES, Schnall MD. MR imaging in patients with nipple discharge: Initial experience. Radiology 2000; 216: 248-54.

2. Vargas HI, Romerol, RTC. Management of bloody nipple discharge. Treat Optn Oncol. 2002; 3: 157-61.

3. Winchester DP. Nipple discharge. In: Diseases of the breast. Haris JR, Lippman ME, Morrow M, Hellman S (eds). Philadelphia, Lippincott, 1996, pp 106-10.

4. Rovno HDS, Slegelman ES, Reynolds C, Orel SC, Schnall MD. Solitary intraductal papilloma: Findings at MR imaging and MR galactography. Am J Roetgenol 1999; 172: 151-55.

5. Piccoli CW, Materucci T, Outwater EK, Siegelman ES, Mitchell DG. Breast carcinoma diagnosis with MR imaging: Effect of clinical and mammographic findings on recommendations for biopsy (abstract). Radiology 1995; 197: 372.

6. Orel SG, Livolsiva S, Troupin RH. Suspicious breast lesions: MR imaging with radiologic pathologic correlation. Radiology 1994; 190: 485-93.

7. Gilles R, Zafrani B, Guinebretire JM, Meuier M, Lucidarme O, Tardivon AA, Rochard F, Vanel D, Neuenschwander S, Arriagada R. Ductal carcinoma in situ MR imaging histopathologic correlation. Radiology 1995; 196: 415-19. 